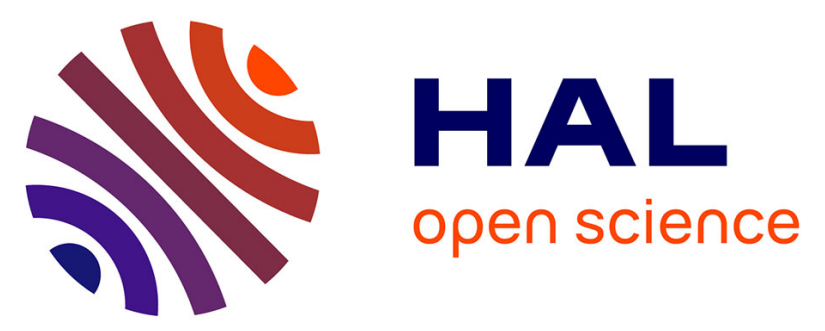

\title{
A Simplified Formulation for Rough Surface Cross-Polarized Backscattering Under the Second-Order Small-Slope Approximation
}

\author{
Charles-Antoine Guérin, Joel T. Johnson
}

\section{- To cite this version: \\ Charles-Antoine Guérin, Joel T. Johnson. A Simplified Formulation for Rough Surface Cross-Polarized Backscattering Under the Second-Order Small-Slope Approximation . IEEE Transactions on Geo- science and Remote Sensing, 2015, 53 (11), pp.6308-6314. 10.1109/TGRS.2015.2440443 . hal- 01171571}

\author{
HAL Id: hal-01171571 \\ https://hal.science/hal-01171571
}

Submitted on 10 Jul 2016

HAL is a multi-disciplinary open access archive for the deposit and dissemination of scientific research documents, whether they are published or not. The documents may come from teaching and research institutions in France or abroad, or from public or private research centers.
L'archive ouverte pluridisciplinaire HAL, est destinée au dépôt et à la diffusion de documents scientifiques de niveau recherche, publiés ou non, émanant des établissements d'enseignement et de recherche français ou étrangers, des laboratoires publics ou privés.

\section{(ㄷ)(i)}

Distributed under a Creative Commons Attribution| 4.0 International License 


\title{
A Simplified Formulation for Rough Surface Cross-Polarized Backscattering Under the Second-Order Small-Slope Approximation
}

\author{
Charles-Antoine Guérin and Joel T. Johnson, Fellow, IEEE
}

\begin{abstract}
We present simplified expressions for the crosspolarized backscatter of a randomly rough surface predicted by the second-order small-slope approximation (SSA2). The simplification is based on appropriate polynomial approximations of the SSA2 kernel function. We obtain numerically efficient expressions for the cross-polarized backscattering amplitude of a deterministic surface in the form of a single space integral involving only the surface elevation and the second (mixed) derivative of the surface elevation. The ensemble average normalized radar cross section is then derived under a Gaussian random process assumption for the surface. The resulting expression has the form of a Kirchhoff integral involving the roughness correlation function and its second- and fourth-order cross-derivatives. Further simplification is achieved for off-nadir observations using a high-frequency approximation; the result is an analytical formula involving only the resonant curvature and the radar-filtered mean square slope in the out-of-plane direction. A numerical validation of the simplified expressions is provided by comparison with exact SSA2 predictions in representative test cases. The dependence of cross-polarized backscattering on the incidence angle as well as wind speed and direction is then investigated for the case of a directional sea surface model. At near nadir incidence, a clear maximum in azimuth of the cross-polarized backscatter is observed for radar look directions $45^{\circ}$ from the wind direction.
\end{abstract}

Index Terms-Cross-polarization, microwave remote sensing, sea surface scattering.

\section{INTRODUCTION}

$\mathbf{T}$ HE use of the cross-polarized backscattering coefficient of the ocean is of increasing recent interest, as it has been found to be a useful proxy for wind speed, especially in high sea states [1]-[3]. However, the interpretation and modeling of rough surface cross-polarized backscattering is still challenging as it involves multiple scattering as well as out-of-plane tilting effects which cannot be simultaneously accounted for by simple analytical models [4]. Today, one of the few scattering models capable of predicting cross-polarized backscatter is the second-order small-slope approximation (SSA2) [5]-[8].

C.-A. Guárin is with the Université de Toulon at the Mediterranean Institute of Oceanography (MIO, UM 110 UTLN/AMU/CNRS-INSU/IRD), 83957 83957 La Garde, France (e-mail: guerin@univ-tln.fr).

J. T. Johnson is with the Department of Electrical and Computer Engineering, The Ohio State University, Columbus, OH 43210 USA.
Computation of SSA2 cross-polarized backscatter predictions, however, remains difficult and computationally demanding because it requires integrations in both the space (over the surface correlation function) and wavenumber (over the surface power spectrum multiplied with an SSA2 "kernel" function) domains, with a slowly decreasing and oscillating integrand in space.

One decade ago, it was shown that the copolarized computation of the SSA2 can be drastically simplified in the so-called high-frequency approximation [9], under which a quadratic approximation of the SSA2 kernel function makes it possible to perform the integration in wavenumber analytically. In this paper, we pursue a similar approach for cross-polarized backscattering to again obtain an approximation of the full SSA2 that involves only a single Kirchhoff-type integral in space. To obtain more accurate predictions, differing approximations are used depending on whether the incidence angle is close to or away from nadir. In the latter case, we further show that an additional high-frequency approximation can be used to reduce the results to an elementary analytical formula involving the surface spectrum at the Bragg resonant wavenumber and the surface mean square slope. We validate the method by comparison with a set of exact SSA2 computations for representative test cases and examine the dependence of the results on wind speed and direction.

This paper is organized as follows. General formulas and notations for the SSA2 technique are recalled in Section II, and the approximation of the SSA2 integral for cross-polarized backscatter is developed in Section III. The corresponding ensemble averaged normalized radar cross section (NRCS) is obtained in Section IV under the assumption of a Gaussian random process surface. High-frequency approximation of the off-nadir NRCS is then described in Section V, and validation tests and numerical illustrations are provided in Section VI.

\section{SSA2 SCATTERING AMPLITUDE}

The sea surface is described by a centered random function $z=\eta(x, y)$ in a Cartesian coordinate system with the $z$-axis directed upward. The surface is illuminated from above by an incident monochromatic plane wave ( $e^{-i \omega t}$ time dependence) with impinging wave vector $\boldsymbol{K}_{0}=\left(\boldsymbol{k}_{0},-q_{0}\right)$ and scattered with outgoing wave vector $\boldsymbol{K}=(\boldsymbol{k},+q)$. The upper medium is air described with vacuum wavenumber $K$. The lower medium is described by a homogeneous complex relative permittivity $\varepsilon$ and a complex wavenumber $K^{\prime}=\sqrt{\varepsilon} K$. The horizontal axis is 
chosen in such a way that the radar look direction is along $x$ $\left(\boldsymbol{k}_{0} \| \boldsymbol{x}\right)$. The SSA2 scattering amplitude [5]-[8] is then

$\mathbb{S}_{2}=\mathbb{S}_{1}-\frac{i}{Q_{z}} \frac{1}{(2 \pi)^{2}} \int d \boldsymbol{r} d \boldsymbol{\xi} e^{-i \boldsymbol{Q}_{\boldsymbol{H}} \cdot r} e^{-i Q_{z} \eta} e^{i \boldsymbol{\xi} \cdot r} \widehat{\eta}(\boldsymbol{\xi}) \mathbb{M}\left(\boldsymbol{k}, \boldsymbol{k}_{0}, \boldsymbol{\xi}\right)$

where $\mathbb{S}_{1}$ is the first-order scattering amplitude (SSA1)

$$
\mathbb{S}_{1}=\frac{1}{Q_{z}} \mathbb{B}_{1} \frac{1}{(2 \pi)^{2}} \int d \boldsymbol{r} e^{-i \boldsymbol{Q}_{\boldsymbol{H}} \cdot r} e^{-i Q_{z} \eta}
$$

and $\widehat{\eta}(\boldsymbol{\xi})$ is the Fourier transform of roughness

$$
\widehat{\eta}(\boldsymbol{\xi})=\frac{1}{(2 \pi)^{2}} \int d \boldsymbol{r} e^{-i \boldsymbol{\xi} \cdot r} \eta(\boldsymbol{r}) .
$$

We have used the standard conventions (e.g., [4]) for the horizontal $\left(\boldsymbol{Q}_{H}=\boldsymbol{k}-\boldsymbol{k}_{0}\right)$ and vertical $\left(Q_{z}=q+q_{0}\right)$ components of the Ewald vector $\boldsymbol{Q}=\boldsymbol{K}-\boldsymbol{K}_{0}$. The involved kernels are, respectively, the first- and second-order Bragg kernels $\mathbb{B}_{1}\left(\boldsymbol{k}, \boldsymbol{k}_{0}\right)$ and $\mathbb{B}_{2}\left(\boldsymbol{k}, \boldsymbol{k}_{0}, \boldsymbol{\xi}\right)$, respectively, and a combination of the latter

$$
\begin{aligned}
\mathbb{M}\left(\boldsymbol{k}, \boldsymbol{k}_{0}, \boldsymbol{\xi}\right)=\frac{1}{2}\left(\mathbb{B}_{2}\left(\boldsymbol{k}, \boldsymbol{k}_{0}, \boldsymbol{k}-\boldsymbol{\xi}\right)\right. & +\mathbb{B}_{2}\left(\boldsymbol{k}, \boldsymbol{k}_{0}, \boldsymbol{k}_{0}+\boldsymbol{\xi}\right) \\
& \left.-2 Q_{z} \mathbb{B}_{1}\left(\boldsymbol{k}, \boldsymbol{k}_{0}\right)\right) .
\end{aligned}
$$

We refer to [7] for the explicit expressions of these kernels (which must be, however, corrected for a conventional factor $q q_{0}$ due to a different definition of the scattering amplitude). One of the difficulties in the numerical computation of exact SSA2 predictions is the presence of terms of the type $q_{\xi}=$ $\sqrt{K^{2}-\xi^{2}}$ and $q_{\xi}^{\prime}=\sqrt{\varepsilon K^{2}-\xi^{2}}$ in the denominator of the kernel $\mathbb{M}$ which produce sharp maxima in wavenumber space.

\section{BACKSCATTERING CROSS-POLARIZED COEFFICIENTS}

We will now focus on the particular but important backscattering configuration $\left(\boldsymbol{k}=-\boldsymbol{k}_{0}, q=q_{0}\right)$. For simplicity, we adopt the notations $M_{12}(\boldsymbol{\xi})$ and $\left(B_{2}\right)_{12}(\boldsymbol{\xi})$ to represent the cross-polarized components of $\mathbb{M}\left(-\boldsymbol{k}_{0}, \boldsymbol{k}_{0}, \boldsymbol{\xi}\right)$ and $\mathbb{B}_{2}\left(-\boldsymbol{k}_{0}\right.$, $\left.\boldsymbol{k}_{0}, \xi\right)$, respectively.

It is well known that the cross-polarized components of the first-order Bragg tensor vanish for backscattering. Hence, the cross-polarized component $\left(S_{2}\right)_{12}$ of SSA2 is merely given by its second-order contribution in (II.1) with

$$
M_{12}(\boldsymbol{\xi})=\frac{1}{2}\left(\left(B_{2}\right)_{12}\left(-\boldsymbol{k}_{0}-\boldsymbol{\xi}\right)+\left(B_{2}\right)_{12}\left(\boldsymbol{k}_{0}+\boldsymbol{\xi}\right)\right) .
$$

The expression for the $B_{2}$ kernel for backscattering is given by ([5] and [7], corrected for a conventional factor $q_{0}^{2}$ )

$$
\begin{aligned}
\left(B_{2}\right)_{12}(\boldsymbol{\xi})= & 2 q_{0} A_{0} \widehat{\boldsymbol{z}}\left[\boldsymbol{\xi}, \widehat{\boldsymbol{k}}_{0}\right] \\
& \times\left(\frac{\varepsilon-1}{\varepsilon q_{\xi}+q_{\xi}} q_{0}^{\prime}\left(\widehat{\boldsymbol{k}}_{0} \cdot \xi\right)-\varepsilon \frac{q_{\xi}+q_{\xi}^{\prime}}{\varepsilon q_{\xi}+q_{\xi}^{\prime}} k_{0}\right)
\end{aligned}
$$

with

$$
A_{0}=\frac{(\varepsilon-1) q_{0} K}{\left(\varepsilon q_{0}+q_{0}^{\prime}\right)\left(q_{0}+q_{0}^{\prime}\right)}
$$

and $z\left[\boldsymbol{\xi}, \widehat{k}_{0}\right]$ denoting the vertical component of the cross product between $\boldsymbol{\xi}$ and $\widehat{k}_{0}$, i.e., $\hat{z} \cdot\left(\boldsymbol{\xi} \times k_{0}\right)$. Due to cancellations when the two $\left(B_{2}\right)_{12}$ terms (which have arguments of equal amplitude but opposite direction) in (III.5) are summed, we obtain

$$
M_{12}(\boldsymbol{\xi})=\frac{2 q_{0} q_{0}^{\prime}(\varepsilon-1) A_{0}}{\varepsilon q_{\left(\boldsymbol{k}_{0}+\boldsymbol{\xi}\right)}+q_{\left(\boldsymbol{k}_{0}+\boldsymbol{\xi}\right)}^{\prime}} \widehat{z}\left[\boldsymbol{\xi}, \widehat{k}_{0}\right]\left(k_{0}+\left(\widehat{\boldsymbol{k}}_{\mathbf{0}} \boldsymbol{\xi}\right)\right) .
$$

To reduce the complexity of the SSA2 integral, we seek an accurate polynomial approximation of this kernel. For this, we distinguish the nadir and off-nadir incidence angles.

\section{A. Off-Nadir Approximation}

The $M_{12}(\boldsymbol{\xi})$ kernel function in (II.1) multiplies $\widehat{\eta}(\boldsymbol{\xi})$ in a Fourier transform over $\boldsymbol{\xi}$. An expansion of $M_{12}(\boldsymbol{\xi})$ that assumes small amplitude for $\boldsymbol{\xi}$ appears appropriate for surfaces whose roughness occurs primarily on length scales long compared to the electromagnetic wavelength (i.e., assuming $\widehat{\eta}(\boldsymbol{\xi})$ is larger for small $\boldsymbol{\xi}$ amplitudes). Due to the known importance of Bragg scattering effects for the sea surface, an approximation of small $\boldsymbol{\xi}$ would seem undesirable in computing off-nadir sea backscatter. However, it will be shown in what follows that this approximation of the kernel function, when applied to the entire range of $\boldsymbol{\xi}$ values, yields an acceptable approximation of the complete SSA2 sea surface cross-polarized backscatter for offnadir angles.

Accordingly, we assume $(\xi \ll K)$ along with $\left\|\boldsymbol{k}_{0}+\boldsymbol{\xi}\right\|<$ $K$, which holds at moderate incidence $\left(k_{0} \ll K\right)$ and sufficiently high wavenumber. Performing a Taylor expansion of the $q_{\boldsymbol{\xi}}$ and $q_{\boldsymbol{\xi}}^{\prime}$ variables about the origin, we obtain

$$
\frac{1}{\varepsilon q_{\left(\boldsymbol{k}_{0}+\boldsymbol{\xi}\right)}+q_{\left(\boldsymbol{k}_{0}+\boldsymbol{\xi}\right)}^{\prime}} \simeq \frac{1}{\varepsilon K+K^{\prime}} \times\left(1+\gamma\left(\frac{1}{2} \frac{k_{0}^{2}}{K^{2}}+\frac{\boldsymbol{k}_{\mathbf{0}} \cdot \xi}{K^{2}}\right)\right)
$$

with

$$
\gamma=\frac{\varepsilon^{3 / 2}+1}{\varepsilon^{3 / 2}+\varepsilon}
$$

This implies that the kernel $M_{12}$ can be approximated by a second-order polynomial of the variable $\xi$

$$
M_{12}(\boldsymbol{\xi}) \simeq \alpha \widehat{z}\left[\boldsymbol{\xi}, \widehat{k}_{0}\right]+\beta \widehat{z}\left[\boldsymbol{\xi}, \widehat{k}_{0}\right]\left(\widehat{\boldsymbol{k}}_{\mathbf{0}} \boldsymbol{\xi}\right)
$$

with

$$
\begin{aligned}
& \alpha=\frac{2 q_{0} q_{0}^{\prime}(\varepsilon-1)}{\varepsilon K+K^{\prime}} A_{0} k_{0}\left(1+\frac{\gamma k_{0}^{2}}{2 K^{2}}\right) \\
& \beta=\frac{2 q_{0} q_{0}^{\prime}(\varepsilon-1)}{\varepsilon K+K^{\prime}} A_{0}\left(1+\frac{3 \gamma k_{0}^{2}}{2 K^{2}}\right) .
\end{aligned}
$$

The first term in (III.11) is linear in $\boldsymbol{\xi}$. Any linear term in $\boldsymbol{\xi}$ in the SSA2 integral (II.1) corresponds to a gradient of roughness

$$
\nabla \eta(\boldsymbol{r})=i \int d \boldsymbol{\xi} e^{i \boldsymbol{\xi} \cdot r} \boldsymbol{\xi} \widehat{\eta}(\boldsymbol{\xi}) .
$$


Any gradient of roughness involved in the Kirchhoff integral can be integrated by parts

$$
\int(\boldsymbol{\nabla} \eta) e^{-i \boldsymbol{Q}_{\boldsymbol{H}} \cdot r} e^{-i Q_{z} \eta}=-\frac{\boldsymbol{Q}_{H}}{Q_{z}} \int e^{-i \boldsymbol{Q}_{\boldsymbol{H}} \cdot r} e^{-i Q_{z} \eta} .
$$

Hence, $\widehat{z}\left[\boldsymbol{\xi}, \widehat{k}_{0}\right]$ can be replaced by $\widehat{z}\left[\left(\boldsymbol{Q}_{H} / Q_{z}\right), \widehat{k}_{0}\right]=0$, so that there is no contribution of the $\alpha$ term of the kernel to the Kirchhoff integral.

The leading term is therefore the $\beta$ term. Without loss of generality, we can assume that $\boldsymbol{k}_{0}$ is directed along the $x$-axis, i.e., $\widehat{k}_{0}=\widehat{x}$, so that the quadratic factor in the cross-polarization kernel reduces to

$$
\widehat{z}\left[\boldsymbol{\xi}, \widehat{k}_{0}\right]\left(\widehat{\boldsymbol{k}}_{\mathbf{0}} \cdot \xi\right)=-\xi_{x} \xi_{y}
$$

Using this approximation, the integration over $\boldsymbol{\xi}$ in (II.1) can now be performed following (III.13), and the result is inserted into (II.1) to obtain

$$
\left(S_{2}\right)_{12}=G_{\gamma} \frac{1}{(2 \pi)^{2}} \int d \boldsymbol{r} \partial_{x y} \eta(\boldsymbol{r}) e^{-i \boldsymbol{Q}_{\boldsymbol{H}} \cdot r} e^{-i Q_{z} \eta}
$$

with

$$
G_{\gamma}=-i \frac{(\varepsilon-1)^{2}}{\varepsilon+\sqrt{\varepsilon}} \frac{q_{0} q_{0}^{\prime}}{\left(\varepsilon q_{0}+q_{0}^{\prime}\right)\left(q_{0}+q_{0}^{\prime}\right)}\left(1+\frac{3 \gamma k_{0}^{2}}{2 K^{2}}\right) .
$$

Note that, in the limit of a perfectly conducting surface $(\varepsilon \rightarrow$ $+\infty)$, this coefficient reduces to

$$
G_{1}=-i\left(1+\frac{3 k_{0}^{2}}{2 K^{2}}\right) .
$$

\section{B. Nadir Case}

For near-nadir angles where the condition $\xi \ll k_{0}$ is no longer valid, it is difficult to obtain a polynomial approximation for the kernel $M_{12}$. Instead, we use the approximation

$$
\frac{1}{\varepsilon q_{\left(\boldsymbol{k}_{0}+\boldsymbol{\xi}\right)}+q_{\left(\boldsymbol{k}_{0}+\boldsymbol{\xi}\right)}^{\prime}} \simeq \frac{1}{\varepsilon q_{\xi}+q_{\xi}^{\prime}}
$$

which is exact for $k_{0}=0$ (nadir incidence). We then obtain

$$
M_{12}(\boldsymbol{\xi}) \simeq \frac{2 q_{0} q_{0}^{\prime}(\varepsilon-1) A_{0}}{\varepsilon q_{\xi}+q_{\xi}^{\prime}} \widehat{z}\left[\boldsymbol{\xi}, \widehat{k}_{0}\right]\left(\widehat{\boldsymbol{k}}_{0} \boldsymbol{\xi}\right) .
$$

This expression is similar to the previous polynomial expression (III.11) with $\gamma=0$ and $\alpha=0$. However, the presence of $q_{\xi}$ and $q_{\xi}^{\prime}$ in the denominator complicates the integration over $\xi$. This issue can be addressed by introducing a modified roughness function $\tilde{\eta}$ defined by the Fourier transform

$$
\widehat{\tilde{\eta}}(\boldsymbol{\xi})=\frac{\varepsilon K+K^{\prime}}{\varepsilon q_{\xi}+q_{\xi}^{\prime}} \widehat{\eta}(\boldsymbol{\xi}) .
$$

Using the modified roughness, the cross-polarized scattering amplitude is found analogously to the previous case as

$$
\left(S_{2}\right)_{12}=G_{0} \frac{1}{(2 \pi)^{2}} \int d \boldsymbol{r} \partial_{x y} \tilde{\eta}(\boldsymbol{r}) e^{-i \boldsymbol{Q}_{\boldsymbol{H}} \cdot r} e^{-i Q_{z} \eta}
$$

where $G_{0}$ is equal to $G_{\gamma}$ in (III.16) with $\gamma=0$ (e.g., in the perfectly conducting case, $G_{0}=-i$ ). Both approximations (III.19) and (III.22) are exact at nadir $\left(k_{0}=0\right)$.

\section{STATISTICAL EXPRESSION}

The preceding section provided formulations for a deterministic surface. We now compute the ensemble average crosspolarized backscattering cross section assuming a Gaussian random process surface, both for the nadir and nonnadir cases.

\section{A. Off-Nadir Case}

Using standard techniques, we obtain

$$
\begin{aligned}
\sigma_{12, \text { off } f \text { ad }}= & \left|G_{\gamma}\right|^{2} \frac{1}{\pi} \int d \boldsymbol{r} e^{-i \boldsymbol{Q}_{\boldsymbol{H}} \cdot r} \\
& \times\left\langle\partial_{x y} \eta(\boldsymbol{r}) \partial_{x y} \eta(\mathbf{0}) e^{-i Q_{z}(\eta(\boldsymbol{r})-\eta(\mathbf{0}))}\right\rangle
\end{aligned}
$$

where the brackets $\langle\star\rangle$ denote the ensemble average. Standard manipulations on Gaussian processes (see the Appendix) lead to

$$
\begin{aligned}
& \left\langle\partial_{x y} \eta(\boldsymbol{r}) \partial_{x y} \eta(\mathbf{0}) e^{-i Q_{z}(\eta(\boldsymbol{r})-\eta(\mathbf{0}))}\right\rangle \\
& =\left(\partial_{x x y y} \rho(\boldsymbol{r})+Q_{z}^{2}\left[\partial_{x y} \rho(\mathbf{0})-\partial_{x y} \rho(\boldsymbol{r})\right]^{2}\right) e^{-Q_{z}^{2}(\rho(0)-\rho(\boldsymbol{r}))}
\end{aligned}
$$

for this correlator, where $\rho$ is the autocorrelation function of elevation

$$
\rho(\boldsymbol{r})=\langle\eta(\boldsymbol{r}) \eta(0)\rangle
$$

that is the inverse Fourier transform of the power spectrum of elevation

$$
\rho(\boldsymbol{r})=\int d \boldsymbol{\xi} \Gamma(\boldsymbol{\xi}) e^{-i \boldsymbol{k} \cdot \xi} .
$$

The cross-polarized off-nadir cross section therefore takes the final form

$$
\begin{array}{r}
\sigma_{12, \text { off } f a d}=\left|G_{\gamma}\right|^{2} \frac{1}{\pi} \int d \boldsymbol{r} e^{-i \boldsymbol{Q}_{\boldsymbol{H}} \cdot r} e^{-Q_{z}^{2}(\rho(\mathbf{0})-\rho(\boldsymbol{r}))} \\
\times\left(\partial_{x x y y} \rho(\boldsymbol{r})+Q_{z}^{2}\left[\partial_{x y} \rho(\mathbf{0})-\partial_{x y} \rho(\boldsymbol{r})\right]^{2}\right) .
\end{array}
$$

\section{B. Nadir Case}

The derivation of the NRCS from the scattering amplitude (III.22) is analogous

$$
\begin{aligned}
\sigma_{12}=\left|G_{0}\right|^{2} & \frac{1}{\pi} \int d \boldsymbol{r} e^{-i \boldsymbol{Q}_{\boldsymbol{H}} \cdot r} \\
& \times\left\langle\partial_{x y} \tilde{\eta}(\boldsymbol{r}) \partial_{x y} \tilde{\eta}(\mathbf{0}) e^{-i Q_{z}(\eta(\boldsymbol{r})-\eta(\mathbf{0}))}\right\rangle .
\end{aligned}
$$

The correlator $\langle\star\rangle$ is now given (see the Appendix) by

$$
\left(\partial_{x x y y} \tilde{\tilde{\rho}}(\boldsymbol{r})+Q_{z}^{2}\left|\partial_{x y} \tilde{\rho}(\mathbf{0})-\partial_{x y} \tilde{\rho}(\boldsymbol{r})\right|^{2}\right) \times e^{-Q_{z}^{2}(\rho(0)-\rho(\boldsymbol{r}))}
$$


where $\tilde{\tilde{\rho}}$ is the autocorrelation function associated to the modified roughness $\tilde{\eta}$ and $\tilde{\rho}$ is the cross-covariance function of $\eta$ and $\tilde{\eta}$

$$
\begin{aligned}
& \tilde{\tilde{\rho}}(\boldsymbol{r})=\int d \boldsymbol{\xi}\left|\frac{\varepsilon K+K^{\prime}}{\varepsilon q_{\boldsymbol{\xi}}+q_{\boldsymbol{\xi}}^{\prime}}\right|^{2} \Gamma(\boldsymbol{\xi}) e^{-i \boldsymbol{k} \cdot \boldsymbol{\xi}} \\
& \tilde{\rho}(\boldsymbol{r})=\int d \boldsymbol{\xi} \frac{\varepsilon K+K^{\prime}}{\varepsilon q_{\boldsymbol{\xi}}+q_{\boldsymbol{\xi}}^{\prime}} \Gamma(\boldsymbol{\xi}) e^{-i \boldsymbol{k} \cdot \boldsymbol{\xi}} .
\end{aligned}
$$

Note that $\tilde{\rho}$ is complex. Altogether, this leads to the following expression for the cross-polarized cross section at nadir:

$$
\begin{aligned}
\sigma_{12, \text { nad }} & =\left|G_{0}\right|^{2} \frac{1}{\pi} \int d \boldsymbol{r} e^{-i \boldsymbol{Q}_{\boldsymbol{H}} \cdot r} e^{-Q_{z}^{2}(\rho(\mathbf{0})-\rho(\boldsymbol{r}))} \\
& \times\left(\partial_{x x y y} \tilde{\tilde{\rho}}(\boldsymbol{r})+Q_{z}^{2}\left|\partial_{x y} \tilde{\rho}(\mathbf{0})-\partial_{x y} \tilde{\rho}(\boldsymbol{r})\right|^{2}\right) .
\end{aligned}
$$

\section{General Case}

The previous sections provide approximations for the nearnadir and off-nadir regions. We propose a combination of these approximations for use at general angles as

$$
\sigma_{12}=\sigma_{12, \text { nad }} \exp \left(-a \tan ^{2} \theta\right)+\left(1-\exp \left(-a \tan ^{2} \theta\right)\right) \sigma_{12, o f f \text { f ad }}
$$

for a specified $a$ which controls the angular width of the transition region between the two approximations. In the numerical results shown, we have chosen the value $a=25$ which limits the nadir correction to about $20^{\circ}$. The expressions for $\sigma_{12, \text { nad }}$ and $\sigma_{12, o f f n a d}$ can be evaluated numerically at the cost of a single Kirchhoff integral, as opposed to the previous fourfold integration required for the SSA2. This simplification is the main result of this paper.

\section{OFF-NADIR High-FrequenCy APPROXIMATION}

Further simplification can be achieved in the high-frequency regime (that is for large $K$ ) in the off-nadir domain using a technique which was first introduced in [10] and termed the "Kirchhoff filtering formula." The squared term in (IV.24) has a quadratic dependence $\sim r^{2}$ about the origin, while $\left|\partial_{x x y y} \rho\right|$ is maximum at zero. At high frequency where the effective integration domain is a small interval around zero, the former term is thus negligible, and it will be ignored in the integral (IV.27). This is confirmed by numerical evidence. Now denote

$$
F(\boldsymbol{r})=e^{-Q_{z}^{2}\left(\rho_{0}-\rho(\boldsymbol{r})\right)} .
$$

Then, by the convolution theorem, we may rewrite

$$
\sigma_{12}=4 \pi\left|G_{\gamma}\right|^{2} \int d \xi_{x} d \xi_{y} \widehat{F}\left(\boldsymbol{Q}_{H}-\boldsymbol{\xi}\right) \xi_{x}^{2} \xi_{y}^{2} \Gamma\left(\xi_{x}, \xi_{y}\right)
$$

or equivalently

$$
\sigma_{12}=4 \pi\left|G_{\gamma}\right|^{2} \int d \xi_{x} d \xi_{y} \widehat{F}\left(\boldsymbol{Q}_{H}-\boldsymbol{\xi}\right) \frac{\xi_{x}^{2}}{\xi^{2}} \frac{\xi_{y}^{2}}{\xi^{2}} B\left(\xi_{x}, \xi_{y}\right)
$$

where $B(\boldsymbol{\xi})=\xi^{4} \Gamma(\boldsymbol{\xi})$ is the curvature spectrum. The function $\widehat{F}$ is a Kirchhoff integral, hence a positive and rapidly decreasing function with its maximum at the origin which acts as a sharp filter around the Bragg frequency $Q_{H}$. To push the calculation further, we approximate the function $F$ by a Gaussian shape $F_{0}$ about the origin

$$
F(\boldsymbol{r}) \simeq F_{0}(\boldsymbol{r})=\exp \left(-\frac{1}{2}\left(\operatorname{mss}_{x} x^{2}+\operatorname{mss}_{y} y^{2}\right)\right)
$$

where the shape parameters $\mathrm{mss}_{x / y}$ are optimized in order to provide a good match of the respective Fourier transforms $\widehat{F}(\boldsymbol{\xi})$ and $\widehat{F}_{0}(\boldsymbol{\xi})$ at $\boldsymbol{\xi}=\boldsymbol{Q}_{H}$. As it is well known from the geometrical optics approximation, the shape parameters are on the order of the radar-filtered directional mss

$$
\operatorname{mss}_{x / y}=\int_{\xi \leq K} d \xi_{x} d \xi_{y} \xi_{x / y}^{2} \Gamma(\boldsymbol{\xi}) .
$$

On the other hand, $B(\boldsymbol{\xi}), \xi_{x}^{2}$, and $\xi^{2}$ are slowly varying functions which can thus be approximated by their value at $\left(\xi_{x}, \xi_{y}\right)=\left(Q_{H}, 0\right)$ in the integral, provided the filter is sharp enough to cutoff spatial frequencies which are below the spectral peak wavenumber. We therefore have the following approximation:

$$
\sigma_{12} \sim 4 \pi\left|G_{\gamma}\right|^{2} Q_{H}^{-2} B\left(\boldsymbol{Q}_{H}\right) \int d \xi_{x} d \xi_{y} \widehat{F}_{0}\left(\boldsymbol{Q}_{H}-\boldsymbol{\xi}\right) \xi_{y}^{2}
$$

obtained by operating the replacements $\xi_{x}^{2} / \xi^{2} \rightarrow 1$ and $\xi_{y}^{2} / \xi^{2} \rightarrow \xi_{y}^{2} / Q_{H}^{2}$ in the previous integral. Since $\boldsymbol{Q}_{\boldsymbol{H}} \cdot \boldsymbol{y}=0$, we have with a simple change of variables $\boldsymbol{\xi} \rightarrow \boldsymbol{Q}_{H}-\boldsymbol{\xi}$

$$
\sigma_{12}=4 \pi\left|G_{\gamma}\right|^{2} Q_{H}^{-2} B\left(\boldsymbol{Q}_{H}\right) \int d \xi_{x} d \xi_{y} \widehat{F}_{0}(\boldsymbol{\xi}) \xi_{y}^{2}
$$

which leads in the end to

$$
\sigma_{12}=4 \pi\left|G_{\gamma}\right|^{2} \operatorname{cotan}^{2}\left(\theta_{i}\right) Q_{H}^{4} \Gamma\left(\boldsymbol{Q}_{H}\right) \mathrm{mss}_{y}
$$

where $\mathrm{mss}_{y}$ is the radar-filtered mean square slope in the direction perpendicular to the incident wave direction. The simple formula (IV.40) is the second main result of this paper. The explicit appearance of the cross slope shows the importance of the out of the incidence plane tilting in the generation of crosspolarized power. The result represents a combination of Bragg scatter effects [due to the presence of $\Gamma\left(\boldsymbol{Q}_{H}\right)$ ] and "long wave tilting" (due to the presence of $\mathrm{mss}_{y}$ ) and can be interpreted as an approximation of the "two-scale" theory of sea surface scattering in which it is assumed that long wave slopes are small and that the sea curvature spectrum is slowly varying in the vicinity of the Bragg wavenumber. Upon inspection of the different approximations which have been used in deriving this simple analytical formula, we see that it is expected to hold in the high-frequency regime (that is for large Rayleigh parameter $\left.Q_{z} \sqrt{\rho_{0}}\right)$ and at off-nadir incidence. 


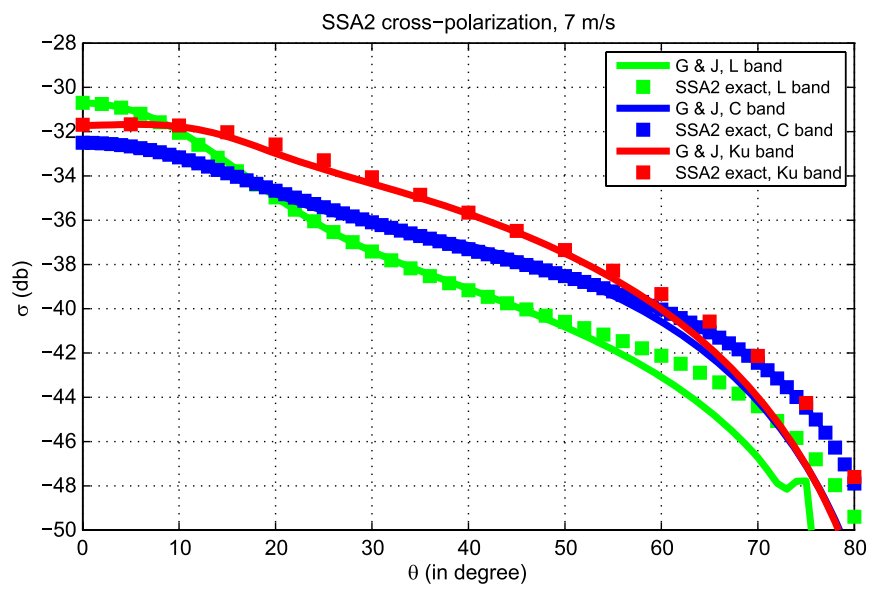

Fig. 1. Approximated SSA2 cross-polarized NRCS according to formula (IV.32) (G\&J) and comparison with the exact SSA2 at different EM bands. The sea state is described by an isotropic Elfouhaily spectrum at $7 \mathrm{~m} / \mathrm{s}$.

\section{Numerical Tests}

The simplified formula (IV.32) has been implemented and compared with an exact SSA2 computation in the case of an Elfouhaily sea surface spectrum [11]. The technique of implementation of the full SSA2 integral has been described in detail elsewhere [12]. The particular method used for the full SSA2 computation requires increasing memory storage as the spatial integral size increases (that is wind speed) and requires sampling on the scale of the electromagnetic wavelength. Therefore, the comparisons were limited to moderate wind speeds. For example, with a 64-GB-RAM computer, we could run $7-\mathrm{m} / \mathrm{s}$ wind speed in $\mathrm{L}, \mathrm{C}$, and $\mathrm{Ku}$ bands. Using the maximum available memory and a much longer computational time, we pushed the calculation to $5-\mathrm{m} / \mathrm{s}$ wind speed in Ka band and also ran a $10-\mathrm{m} / \mathrm{s}$ wind speed case at $\mathrm{X}$ band.

\section{A. Isotropic Spectra}

Fig. 1 compares the full and simplified SSA2 cross-polarized NRCS versus incidence angle for an isotropic Elfouhaily spectrum at $7-\mathrm{m} / \mathrm{s}$ wind speed. $\mathrm{L}$ band $\left(\lambda_{E M}=23.8 \mathrm{~cm}, \varepsilon=\right.$ $75+i 61), \mathrm{C}$ band $\left(\lambda_{E M}=6 \mathrm{~cm}, \varepsilon=67+i 36\right)$, and $\mathrm{Ku}$ band $\left(\lambda_{E M}=2.143 \mathrm{~cm}, \varepsilon=42+i 39.5\right)$ frequencies are included. The agreement is found excellent over a wide range of incidence angles spanning from nadir to about $60^{\circ}$.

Fig. 2 illustrates similar comparisons for an isotropic Elfouhaily spectrum at $10-\mathrm{m} / \mathrm{s}$ wind speed in $\mathrm{X}$ band $\left(\lambda_{E M}=\right.$ $3 \mathrm{~cm}, \varepsilon=60.63+i 44.97)$. To clarify the respective contributions of the "nadir" and "off nadir" parts, we have plotted the results of formulas (IV.31) and (IV.27) separately and show that an appropriate combination of both is necessary to remain accurate over a wide range of incidence angles.

Fig. 3 shows the high-frequency off-nadir approximation (V.40) for the $\mathrm{L}, \mathrm{Ku}$, and $\mathrm{Ka}$ bands $\left(\lambda_{E M}=8 \mathrm{~mm}, \varepsilon=15+\right.$ i26) for the same spectrum at $7 \mathrm{~m} / \mathrm{s}$ ( $\mathrm{L}$ and $\mathrm{Ku}$ bands) and $5 \mathrm{~m} / \mathrm{s}$ (Ka band). The exact SSA2 calculation is superimposed. The analytical approximation of SSA2 is found in overall very good agreement with the latter beyond $30^{\circ}$ incidence and is increasingly accurate at higher radar frequencies.

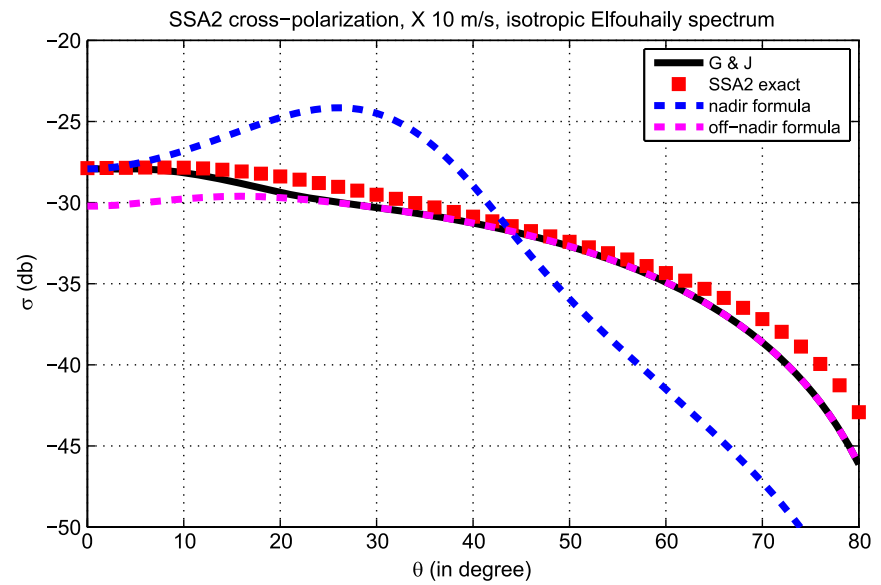

Fig. 2. Approximated SSA2 cross-polarized NRCS according to formulas (IV.32) (G\&J) and comparison with the exact SSA2 in X band. Superimposed are the contributions of the nadir (IV.31) and off-nadir (IV.27) formulas. The sea state is described by an isotropic Elfouhaily spectrum at $10 \mathrm{~m} / \mathrm{s}$.

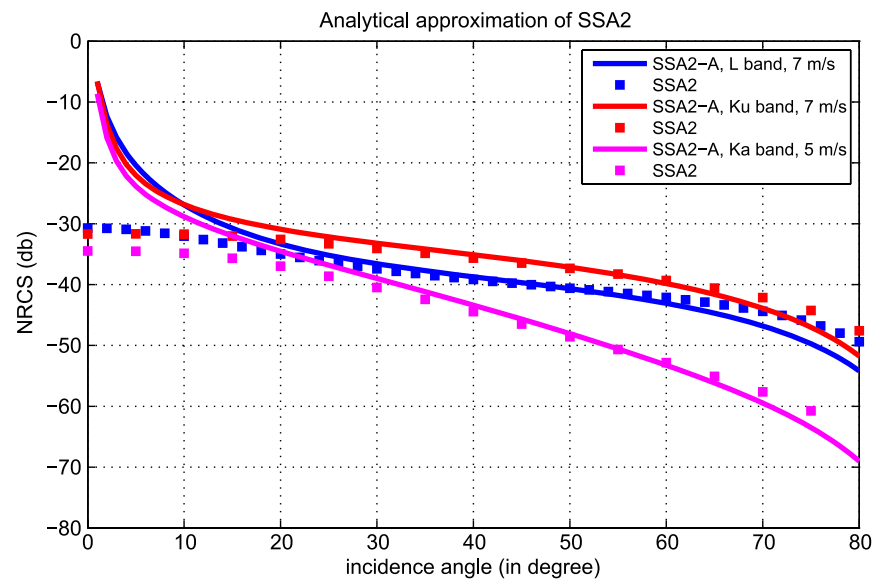

Fig. 3. Approximation of SSA2 cross-polarized NRCS according to the analytical approximation (V.40) (SSA2-A) and comparison with the exact SSA2 in different bands. The sea state is described by an isotropic Elfouhaily spectrum at $7 \mathrm{~m} / \mathrm{s}$ in $\mathrm{L}$ and $\mathrm{Ku}$ bands and $5 \mathrm{~m} / \mathrm{s}$ in $\mathrm{Ka}$ band.

\section{B. Anisotropic Spectra}

The dependence on both wind speed and direction has also been investigated with a directional Elfouhaily spectrum (see the Appendix). A comparison with the exact SSA2 calculations presented in [8] is displayed in Fig. 4 for a wind speed of $15 \mathrm{~m} / \mathrm{s}$ in X band. The method of performing the exact SSA2 computations in this reference allows higher wind speeds to be considered. A good agreement is found both at nadir and larger incidence angles with a maximum of $1-\mathrm{dB}$ error at intermediate incidence angles.

Fig. 5 plots the predicted NRCS as a function of wind speed for the upwind, crosswind, and $45^{\circ}$ azimuth direction for both nadir and $45^{\circ}$ incidence angle. At nadir, a maximum of the cross-polarized NRCS is found for azimuth angle $45^{\circ}$ with respect to the radar polarization vector. At $45^{\circ}$ incidence, the qualitative behaviors are similar to the copolarized case, with a maximum in up/downwind direction and a minimum in crosswind direction. 


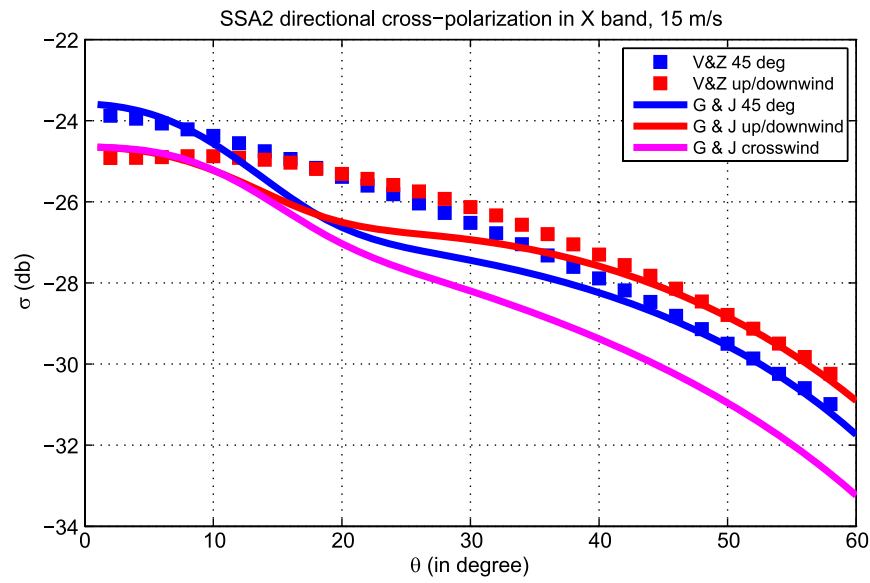

Fig. 4. Approximated SSA2 cross-polarized NRCS according to formula (IV.32) (G \& J) and comparison with the exact SSA2 X band data from [8] (V \& Z, courtesy of V. Zavorotny and A. Voronovich). The sea state is described by a directional Elfouhaily spectrum at $15 \mathrm{~m} / \mathrm{s}$.

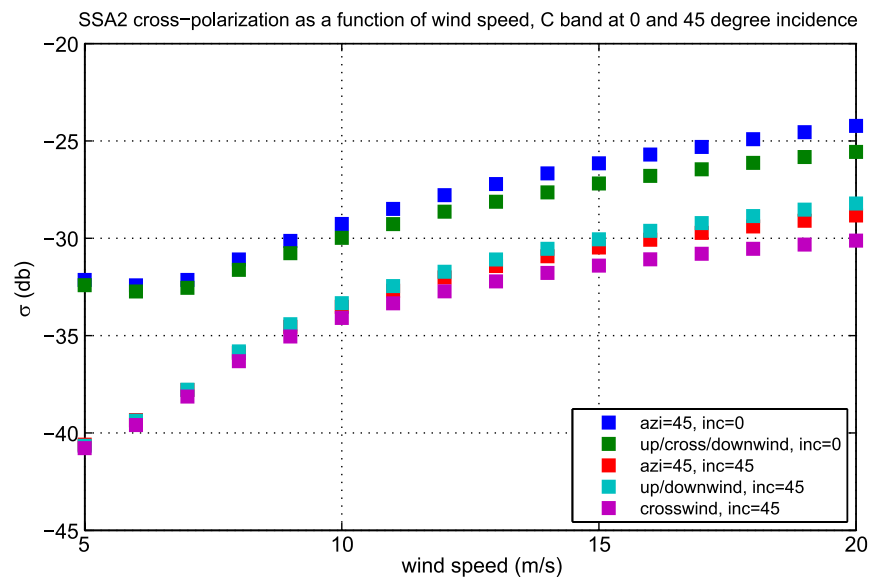

Fig. 5. Approximated SSA2 cross-polarized NRCS according to formula (IV.32) $\left(\mathrm{G} \mathrm{\&} \mathrm{J)} \mathrm{as} \mathrm{a} \mathrm{function} \mathrm{of} \mathrm{wind} \mathrm{speed} \mathrm{for} \mathrm{two} \mathrm{incidences}\left(0\right.\right.$ and $\left.45^{\circ}\right)$. The sea state is described by a directional Elfouhaily spectrum.

\section{CONCLUSION}

We have derived a simplified formulation for rough surface cross-polarized backscattering under the SSA2 analytical model. This makes the SSA2 more tractable for numerical applications, at the price of an approximation which has been found reasonable for a variety of wind speeds and electromagnetic frequencies at nongrazing incidence angles, particularly for near-nadir and moderately large incidence angles of $30-60^{\circ}$. For Ka band frequencies and higher, an additional approximation was found, which involved only the resonant Bragg frequency of the sea surface spectrum and its cross-plane mss, which unveils the specific contribution of the out-of-plane tilting in the cross-polarization mechanism away from nadir. Further tests and comparisons are necessary to fully assess these first findings, and they will be continued in the future. Although the examples illustrated focused on cross-polarized backscatter from the sea surface, the simplified formulas developed can also be applied for other surface types and in other applications.

\section{APPENDIX}

\section{A. Calculation of the Correlators}

To obtain (IV.29) (as well as (IV.24) which is a particular case), we proceed in the following way. We consider the fourpoint characteristic function

$$
\mathcal{C}(\alpha, \beta)=\left\langle e^{-i Q_{z}\left(\eta-\eta_{0}\right)+i \alpha \partial_{x y} \tilde{\eta}+i \beta \partial_{x y} \tilde{\eta}_{0}}\right\rangle
$$

where the dependence on position $r$ is implicit and the 0 subscript refers to $r=0$. We observe that the correlator (IV.29) is given by

$$
-\left.\partial_{\alpha \beta} \mathcal{C}(\alpha, \beta)\right|_{\alpha=\beta=0} .
$$

Since the roughness processes $\eta$ and $\tilde{\eta}$ are Gaussian, this amounts to evaluating

$$
\mathcal{C}(\alpha, \beta)=e^{-\frac{1}{2}\left\langle\left|Q_{z}\left(\eta-\eta_{0}\right)-\alpha \partial_{x y} \tilde{\eta}-\beta \partial_{x y} \tilde{\eta}_{0}\right|^{2}\right\rangle}
$$

which involves the roughness autocorrelation function (IV.25) as well as the modified roughness autocorrelation function $\tilde{\tilde{\rho}}(\boldsymbol{r})=\left\langle\tilde{\eta}^{*}(\boldsymbol{r}) \tilde{\eta}(0)\right\rangle$ and the cross-correlation function $\tilde{\rho}(\boldsymbol{r})=$ $\left\langle\tilde{\eta}^{*}(\boldsymbol{r}) \eta(0)\right\rangle$ (the latter can be complex). Straightforward calculations then lead to the expression (IV.29).

\section{B. Implementation of the Kirchhoff Integrals}

We propose an efficient numerical implementation of the integral (IV.27) in the case of biharmonic spectra of the form

$$
\Gamma(\boldsymbol{k})=\Gamma(k, \varphi)=\Gamma_{0}(k)+\Gamma_{2}(k) \cos \left(2 \varphi-2 \varphi_{w}\right)
$$

where $\varphi_{k}$ is the angle of the vector $\boldsymbol{k}$ with respect to the incident wave vector direction (which is taken to be the $\widehat{\boldsymbol{x}}$ axis) and $\varphi_{w}$ is the angle of the wind vector with respect to the same reference. The implementation of the integral (IV.31) is similar when using the modified spectra described in Section IV-B.

We denote by $\mathcal{B}_{n}[f](u)$ the Bessel transform of a function $f$ at $n$th order, which is explicitly

$$
\mathcal{B}_{n}[\Gamma](r)=\int_{0}^{\infty} d k j_{n}(k r) 2 \pi k \Gamma(k)
$$

or

$$
\mathcal{B}_{n}[F]\left(Q_{H}\right)=\int_{0}^{\infty} d r j_{n}\left(Q_{H} r\right) 2 \pi r F(r)
$$

the integration being performed with respect to the space or wavenumber variable according to the function to which it is applied. For the following, we recall the useful identities:

$$
\begin{aligned}
& \frac{\left(i^{-n}\right)}{2 \pi} \int_{0}^{2 \pi} d \varphi \cos (n \varphi) e^{i u \cos \left(\varphi-\varphi_{0}\right)}=\cos \left(n \varphi_{0}\right) j_{n}(u) \\
& \frac{\left(i^{-n}\right)}{2 \pi} \int_{0}^{2 \pi} d \varphi \sin (n \varphi) e^{i u \cos \left(\varphi-\varphi_{0}\right)}=\sin \left(n \varphi_{0}\right) j_{n}(u) .
\end{aligned}
$$


It is then straightforward to derive the following expressions (with $x=r \cos \varphi_{r}, y=r \sin \varphi_{r}$ ):

$$
\begin{aligned}
\rho= & \mathcal{B}_{0}\left[\Gamma_{0}\right](r)-\mathcal{B}_{2}\left[\Gamma_{2}\right](r) \cos \left(2 \varphi_{r}-2 \varphi_{w}\right) \\
\partial_{x y} \rho= & \frac{1}{2} \mathcal{B}_{2}\left[k^{2} \Gamma_{0}\right](r) \sin \left(2 \varphi_{r}\right) \\
& -\frac{1}{4} \mathcal{B}_{0}\left[k^{2} \Gamma_{2}\right](r) \sin \left(2 \varphi_{w}\right) \\
& -\frac{1}{4} \mathcal{B}_{4}\left[k^{2} \Gamma_{2}\right](r) \sin \left(4 \varphi_{r}-2 \varphi_{w}\right) \\
\partial_{x x y y} \rho= & \frac{1}{8} \mathcal{B}_{0}\left[k^{4} \Gamma_{0}\right](r)-\frac{1}{8} \mathcal{B}_{4}\left[k^{4} \Gamma_{0}\right](r) \cos \left(4 \varphi_{r}\right) \\
& -\frac{1}{8} \mathcal{B}_{2}\left[k^{4} \Gamma_{2}\right](r) \cos \left(2 \varphi_{r}-2 \varphi_{w}\right) \\
& +\frac{1}{16} \mathcal{B}_{2}\left[k^{4} \Gamma_{2}\right](r) \cos \left(2 \varphi_{r}+2 \varphi_{w}\right) \\
& +\frac{1}{16} \mathcal{B}_{6}\left[k^{4} \Gamma_{2}\right](r) \cos \left(6 \varphi_{r}-2 \varphi_{w}\right) .
\end{aligned}
$$

In the case of isotropic spectrum $\left(\Gamma_{2}=0\right)$, an efficient calculation of the Kirchhoff integral can be obtained using Bessel transforms. Using the identities (A47), we obtain

$$
\begin{aligned}
\int e^{-i \boldsymbol{Q}_{\boldsymbol{H}} \cdot \boldsymbol{r}}\left(\partial_{x x y y} \rho\right) F= & \frac{1}{8} \mathcal{B}_{0}\left[\mathcal{B}_{0}\left[k^{4} \Gamma_{0}\right] F\right]\left(Q_{H}\right) \\
& -\frac{1}{8} \mathcal{B}_{4}\left[\mathcal{B}_{4}\left[k^{4} \Gamma_{0}\right] F\right]\left(Q_{H}\right) \\
\int e^{-i \boldsymbol{Q}_{\boldsymbol{H}} \cdot \boldsymbol{r}}\left(\partial_{x y} \rho\right)^{2} F= & \frac{1}{8} \mathcal{B}_{0}\left[\left(\mathcal{B}_{2}\left[k^{2} \Gamma_{0}\right]\right)^{2} F\right]\left(Q_{H}\right) \\
& -\frac{1}{8} \mathcal{B}_{4}\left[\left(\mathcal{B}_{2}\left[k^{2} \Gamma_{0}\right]\right)^{2} F\right]\left(Q_{H}\right) .
\end{aligned}
$$

In the case of an anisotropic spectrum, the Kirchhoff integral is calculated with integration in polar coordinates using a Simpson quadrature rule to accelerate the angular integration.

\section{REFERENCES}

[1] P. A. Hwang, B. Zhang, and W. Perrie, "Depolarized radar return for breaking wave measurement and hurricane wind retrieval," Geophys. Res. Lett., vol. 37, no. 1, Jan. 2010, Art. ID. L01604.

[2] P. W. Vachon and J. Wolfe, "C-band cross-polarization wind speed retrieval," IEEE Geosci. Remote Sens. Lett., vol. 8, no. 3, pp. 456-459, May 2011.

[3] P. A. Hwang, W. Perrie, and B. Zhang, "Cross-polarization radar backscattering from the ocean surface and its dependence on wind velocity," IEEE Trans. Geosci. Remote Sens., vol. 11, no. 12, pp. 2188-2192, Dec. 2014

[4] T. Elfouhaily and C. A. Guérin, "A critical survey of approximate scattering wave theories from random rough surfaces," Waves Random Complex Media, vol. 14, no. 4, pp. 1-40, 2004.

[5] A. Voronovich, "Small-slope approximation for electromagnetic wave scattering at a rough interface of two dielectric half-spaces," Waves Random Complex Media, vol. 4, no. 3, pp. 337-367, 1994.

[6] A. G. Voronovich, Wave Scattering From Rough Surfaces, ser. Springer Series on Wave Phenomena. New York, NY, USA: Springer-Verlag, 1994.
[7] A. G. Voronovich and V. U. Zavorotny, "Theoretical model for scattering of radar signals in $\mathrm{Ku}$ - and $\mathrm{C}$-bands from a rough sea surface with breaking waves," Waves Random Media, vol. 11, no. 3, pp. 247-269, 2001.

[8] A. Voronovich and V. Zavorotny, "Full-polarization modeling of monostatic and bistatic radar scattering from a rough sea surface," IEEE Trans. Antennas Propag., vol. 62, no. 3, pp. 1363-1371, Mar. 2014.

[9] C. -A. Guérin and M. Saillard, "On the high-frequency limit of the secondorder small-slope approximation," Waves Random Media, vol. 13, no. 2, pp. 75-88, 2003.

[10] C. A. Guérin, G. Soriano, and B. Chapron, "The weighted curvature approximation in scattering from sea surfaces," Waves Random Complex Media, vol. 14, no. 3, pp. 349-363, 2010.

[11] T. Elfouhaily, B. Chapron, K. Katsaros, and D. Vandemark, "A unified directional spectrum for long and short wind-driven waves," J. Geophys. Res., vol. 102, no. C7, pp. 15 781-15 796, Jul. 1997.

[12] T. M. Elfouhaily and J. T. Johnson, "A new model for rough surface scattering," IEEE Trans. Geosci. Remote Sens., vol. 45, no. 7, pp. 2300-2308, Jul. 2007.

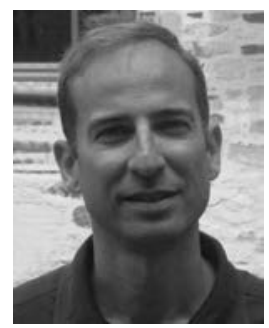

Charles-Antoine Guérin received the B. Eng. degree from Ecole Nationale Supérieure de l'Aéronautique et de l'Espace, Toulouse, France, in 1994 and the Ph.D. degree in theoretical physics from Aix Marseille University, Marseille, France, in 1998.

$\mathrm{He}$ is currently a Professor and a Researcher with the Mediterranean Institute of Oceanography, University of Toulon, La Garde, France. He is specialized in ocean remote sensing.

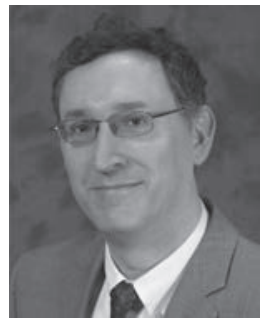

Joel T. Johnson (S'88-M'96-SM'03-F'08) received the B.S. degree in electrical engineering from the Georgia Institute of Technology, Atlanta, GA, USA, in 1991 and the S.M. and Ph.D. degrees from the Massachusetts Institute of Technology, Cambridge, MA, USA, in 1993 and 1996, respectively.

He is currently a Professor and Department Chair with the Department of Electrical and Computer Engineering and ElectroScience Laboratory, The Ohio State University, Columbus, OH, USA. His research interests are in the areas of microwave remote sensing, propagation, and electromagnetic wave theory.

Dr. Johnson is a member of commissions B and F of the International Union of Radio Science (URSI) and a member of Tau Beta Pi, Eta Kappa Nu, and Phi Kappa Phi. He received the 1993 Best Paper Award from the IEEE Geoscience and Remote Sensing Society, was named an Office of Naval Research Young Investigator, National Science Foundation Career awardee, and PECASE award recipient in 1997, and was recognized by the U.S. National Committee of URSI as a Booker Fellow in 2002. 